\title{
Event-Triggered State Estimation for a Class of Delayed Recurrent Neural Networks with Sampled-Data Information
}

\author{
Hongjie Li \\ College of Mathematics, Physics and Information Engineering, Jiaxing University, Zhejiang 314001, China
}

Correspondence should be addressed to Hongjie Li, lhjymlly@163.com

Received 9 June 2012; Accepted 31 July 2012

Academic Editor: Sabri Arik

Copyright (C) 2012 Hongjie Li. This is an open access article distributed under the Creative Commons Attribution License, which permits unrestricted use, distribution, and reproduction in any medium, provided the original work is properly cited.

The paper investigates the state estimation problem for a class of recurrent neural networks with sampled-data information and time-varying delays. The main purpose is to estimate the neuron states through output sampled measurement; a novel event-triggered scheme is proposed, which can lead to a significant reduction of the information communication burden in the network; the feature of this scheme is that whether or not the sampled data should be transmitted is determined by the current sampled data and the error between the current sampled data and the latest transmitted data. By using a delayed-input approach, the error dynamic system is equivalent to a dynamic system with two different time-varying delays. Based on the Lyapunov-krasovskii functional approach, a state estimator of the considered neural networks can be achieved by solving some linear matrix inequalities, which can be easily facilitated by using the standard numerical software. Finally, a numerical example is provided to show the effectiveness of the proposed event-triggered scheme.

\section{Introduction}

The research of neural networks has been paid much attention during the past few years, due to its potential application in various fields, such as image processing, pattern recognition, and associative memory [1-5]. As a special class of nonlinear dynamical systems, the dynamic behavior of recurrent neural networks has been one of the most important issues. In particular, the analysis problems of stability and synchronization of recurrent neural networks have received great attention and a number of profound results have been proposed [6-12].

In many application, such as signal processing and control engineering, for large-scale neural networks, it is quite common that only partial information can be accessible from the 
network outputs. Therefore, it is of great significance to estimate the neuron states through available output measurements of the networks and then utilizes the estimated neuron states to achieve certain design objectives; note that state estimation problem for neural networks has been hot reach topics that have drawn considerable attention, and many profound results have been available in the literature [13-25]. The authors in [13] studied the problem of state estimation for a class of delayed neural networks; the traditional monotonicity and smoothness assumption on the activation function had been removed. The design problem of state estimator for a class of neural networks with constant delays was investigated in [14], where a delay-dependent criterion for existence of the estimator was proposed. As an extension, The authors in $[14,15]$ further discussed state estimation for neural networks with time-varying delays. In practice, sometimes a neural network has finite state modes and modes may switch from one to another at different times. On the other hand, discrete-time neural networks could be more suitable to model digitally transmitted signals in dynamical way; based on the above reason, The authors in [16] investigated state estimation problem for a new class of discrete-time neural networks with Markovian jumping parameters and mode dependent mixed time-delays, where he discrete and distributed delays were modedependent. Different from the stuelies in $[16,17]$ which considered state estimation for Markovian jumping delayed continuous-time recurrent neural networks, where only matrix parameters were mode-dependent. Similar to [16], for continuous-time recurrent neural networks with discrete and distributed delays, state estimation was also investigated in [18]. In $[19,20]$, synchronization and state estimation had been studied for discrete-time complex networks with distributed delays; it was noticed that in [20], a novel notion of bounded $H_{\infty}$ synchronization had been first defined to characterize the transient performance of synchronization. Some robust state estimation problems for uncertain neural networks with time-varying delays had been investigated in [21-23], where the parameter uncertainties are assumed to be norm bounded; some sufficient conditions were presented to guarantee the existence of the desired state estimator. Taking into account the stochastic properties of timevarying delays, the authors in [24] discussed state estimation problem for a class of discretetime stochastic neural networks with random delays; sufficient delay-distribution-dependent conditions were established in terms of linear matrix inequalities (LMIs) that guarantee the existence of the state estimator.

The sampled-data control theory had attracted much attention due to its effectiveness in engineering applications. Especially, a new approach to deal with the sampled-data control problems had been proposed in [26], where the sampling period had been converted into time-varying delay. As its extension, the authors in [27] investigated the sampled-data state estimation problem for a class of recurrent neural networks with time-varying delays, where the sampled measurements had been used to estimate the neuron states. Using a similar approach, where the sampled-data synchronization control problem was investigated in [28] for a class of general delayed complex networks, the sampled-data feedback controllers were designed in terms of the solution to certain linear matrix inequalities. But in the above references, the sampling rate for each signal is the same; but in the actual system, it may be varying from sample to sample owing to unpredictable perturbations; this factor was considered in [29], the problem of robust $H_{\infty}$ control was investigated for sampled-data systems with probabilistic sampling, where two different sampling periods were considered whose occurrence probabilities were given constants and satisfied Bernoulli distribution. In [30], stochastic sampled-data approach was used for studying the problem of distributed $H_{\infty}$ filtering in sensor networks, by converting the sampling periods into bounded time-delays, the design problem of $H_{\infty}$ filters amounted to solving the $H_{\infty}$ filtering problem for a class 
of stochastic nonlinear systems with multiple bounded time delays. In [31], the sampleddata synchronization control problem was addressed, where the sampling period was time varying and switched between two different values in a random way. It is worth noting that most of the above results were involved the traditional approach of sampling at prespecified time instances, which was called time-triggered sampling; this sampling method may lead to an inherently periodic transmission and produce many useless messages if the current sampled signal had not significantly changed in contrast to the previous sampled signal, which led to a conservative usage of the communication resources. Recently, eventtriggered scheme provided an effective approach of determining; its main property was that the signal was sampled and only some functions of the system state or output measurement exceeded threshold. Compared with periodic sampling method, the event-triggered scheme could reduce the burden of the communication and also preserve the desired properties of the ideal continuous state feedback system, such as stability and convergence. The utilization on event-triggered scheme could be found in many literatures such as [32-37]. The eventtriggered $H_{\infty}$ control design was investigated in [32] for networked control systems with uncertainties and transmission delays, and a novel event-triggered scheme was proposed. The study in [33] was concerned with the control problem of event-triggered networked systems with both state and control input quantizations. In [34], the problems of exponential stability and $L_{2}$-gain analysis of event-triggered networked control systems were studied, where the event-triggered conditions were proposed in the sensor side and controller side. In [35-37], the consensus problems for multiagent systems were investigated by event-triggered control, where different trigger functions were proposed. Unfortunately, as far as we know, up to now, no theoretical results are given for state estimation of recurrent neural networks with time-varying delays based on event-triggered scheme. The purpose of our study is to fill the gap.

Motivated by the above discussion, the paper is concerned with the sampled-data state estimation problem for a class of recurrent neural networks with time-varying delays. The main purpose is to estimate the neuron states through output sampled measurement, and a novel event-triggered scheme is proposed, which can lead to a significant reduction of the information communication burden in the network. By using a delayed-input approach, the error dynamics system is equivalent to a dynamic system with two different time-varying delays. Based on constructing a Lyapunov-Krasovskii functional and employing some analysis techniques, a state estimator of the considered neural networks can be achieved by solving some linear matrix inequalities, which can be easily facilitated by using the standard numerical software. Finally, a numerical example is given to illustrate the effectiveness of the proposed method.

The main contributions of this paper are highlighted as follows.

(1) The novel event-triggered scheme is proposed, compared with a time-triggered periodic communication scheme, since the proposed communication scheme only depends on the state at the sampled instant and the state error between the current sampled instant and the latest transmitted state. Therefore, the number of the transmitted state signals through the network could be reduced apparently.

(2) Sufficient conditions obtained are in the form of linear matrix inequalities which can be readily solved by using the LMI toolbox in Matlab, and the solvability of derived conditions dependents on not only trigger parameters and sampling period but also the size of the delay. 
Notation 1. The notation used here is fair standard except where otherwise stated. $\mathbb{R}^{n}$ denotes the $n$-dimensional Euclidean space and $\mathbb{R}^{n \times m}$ is the set of real $n \times m$ matrices. The superscript $T$ represents the transpose of the matrix (or vector). $I$ denotes the identity matrix of compatible dimensions. The asterisk represents the symmetric block in one symmetric matrix. $\operatorname{diag}\{\cdots\}$ stands for a block-diagonal matrix. The notation $X \geq 0(X>0)$ means that $X$ is positive semidefinite (positive definite). $\|\cdot\|$ is the Euclidean norm in $\mathbb{R}^{n}$. If they are not explicitly specified, arguments of a function or a matrix will be omitted in the analysis when no confusion can arise.

\section{Preliminaries}

Consider a class of recurrent neural networks with time-varying delays as follows:

$$
\begin{gathered}
\dot{x}(t)=-A x(t)+W_{0} g(x(t))+W_{1} g(x(t-\tau(t))) \\
y(t)=C x(t)
\end{gathered}
$$

where $x(t)=\left(x_{1}(t), x_{2}(t), \ldots, x_{n}(t)\right)^{T} \in \mathbb{R}^{n}$ is the state vector associated with $n$ neurons; $A=$ $\operatorname{diag}\left\{a_{1}, a_{2}, \ldots, a_{n}\right\}$ is a positive diagonal matrix; $W_{0} \in \mathbb{R}^{n \times n}$ and $W_{1} \in \mathbb{R}^{n \times n}$ are the connection weight matrix and the delayed connection weight matrix, respectively; $\tau(t) \in[0, \tau]$ is the time-varying bound delay; $C \in \mathbb{R}^{m \times n}$ is a constant matrix; $y(t)=\left(y_{1}(t), y_{2}(t), \ldots, y_{m}(t)\right)^{T} \in$ $\mathbb{R}^{m}$ denotes the output vector; $g(x(t))=\left[g_{1}\left(x_{1}(t)\right), g_{2}\left(x_{2}(t)\right), \ldots, g_{n}\left(x_{n}(t)\right)\right]^{T} \in \mathbb{R}^{n}$ represents the neuron activation function with $g(0)=0$.

In this paper, the measurement output is sampled before it enters the estimator; based on the sampling technique and zero-order hold, the actual output can be described as

$$
\bar{y}(t)=y\left(t_{k}\right)=C x\left(t_{k}\right), \quad t \in\left[t_{k}, t_{k+1}\right)
$$

where $\bar{y}(t) \in \mathbb{R}^{m}$ is the actual output of the estimator, and $t_{k}$ denotes the sampling instant satisfying $\lim _{k \rightarrow \infty} t_{k}=\infty$.

Remark 2.1. In practical systems, periodic sampling mechanism may often lead to sending many unnecessary signals through the networks, which will increase the load of network transmission and waste the network bandwidth; therefore, it is significant to introduce a mechanism to determine which sampled signal should be sent out or not. As stated in [32,33], the event-trigger sampling scheme is effective way because they can reduce the traffic and the power consumption.

The sampled data $y\left(t_{k+j}\right)$ is transmitted (or released) by the event generator only when the current sampled value $y\left(t_{k+j}\right)$ and the previously transmitted one $y\left(t_{k}\right)$ satisfy the following judgement algorithm:

$$
\left[y\left(t_{k+j}\right)-y\left(t_{k}\right)\right]^{T} W\left[y\left(t_{k+j}\right)-y\left(t_{k}\right)\right]<\sigma y^{T}\left(t_{k+j}\right) W y\left(t_{k+j}\right)
$$

where $W \in \mathbb{R}^{m \times m}$ is a positive matrix, and $\sigma \in[0,1)$ is a positive scalar. The sampled state $y\left(t_{k+j}\right)$ satisfying the inequality (2.3) will not be transmitted; only the one that exceeds the threshold in (2.3) will be sent to the estimator. Specially, when $\sigma=0$, the inequality (2.3) is 
not satisfied for almost all the sampled state $y\left(t_{k+j}\right)$, and the event-triggered scheme reduces to a periodic release scheme.

Remark 2.2. From event-triggered algorithm (2.3), it is easily seen that all the released signals are subsequences of the sampled data, that is, the set of the release instants $\left\{t_{0}, t_{1}, t_{2} \ldots\right\} \subseteq$ $\{0,1,2, \ldots\}$. The amount of $\left\{t_{0}, t_{1}, t_{2} \ldots\right\}$ depends on not only the value of $\sigma$ but also the variation of the system output.

Suppose that the time-varying delay in network communication is $d_{k} \in[0, d](k=$ $1,2, \ldots,+\infty)$, the output $\bar{y}(t)$ in $(2.2)$ can be rewritten as

$$
\bar{y}(t)=y\left(t_{k}\right)=C x\left(t_{k}\right), \quad t \in\left[t_{k}+d_{k}, t_{k+1}+d_{k+1}\right)
$$

Substituting the output (2.4) into the judgement algorithm (2.3), we can obtain

$$
\left[x\left(t_{k+j}\right)-x\left(t_{k}\right)\right]^{T} C^{T} W C\left[x\left(t_{k+j}\right)-x\left(t_{k}\right)\right]<\sigma x^{T}\left(t_{k+j}\right) C^{T} W C x\left(t_{k+j}\right) .
$$

For technical convenience, similar to $[32,33]$, consider the following two intervals:

$$
\left[t_{k}+d_{k}, t_{k}+h+d\right), \quad\left[t_{k}+l h+d, t_{k}+l h+h+d\right),
$$

where $l$ is a positive integer and $h$ is a sampling period.

(1) if $t_{k}+h+d>t_{k+1}+d_{k+1}$, define a function $d(t)$ as follows:

$$
d(t)=t-t_{k}, \quad t \in\left[t_{k}+d_{k}, t_{k+1}+d_{k+1}\right) .
$$

It can easily be obtained that the following inequality holds:

$$
d_{k} \leq d(t) \leq t_{k+1}-t_{k}+d_{k+1} \leq h+d
$$

(2) if $t_{k}+h+d<t_{k+1}+d_{k+1}$, there exists a positive integer $m$, such that

$$
t_{k}+m h+d<t_{k+1}+d_{k+1}<t_{k}+m h+h+d .
$$

It can be easily shown that

$$
\left[t_{k}+d_{k}, t_{k+1}+d_{k+1}\right)=I_{1} \cup I_{2} \cup I_{3},
$$


where

$$
\begin{gathered}
I_{1}=\left[t_{k}+d_{k}, t_{k}+h+d\right) \\
I_{2}=\bigcup_{m=1}^{l-1}\left\{I_{2}^{m}\right\} \\
I_{2}^{m}=\left[t_{k}+m h+d, t_{k}+m h+h+d\right) \\
I_{3}=\left[t_{k}+l h+d, t_{k+1}+d_{k+1}\right) .
\end{gathered}
$$

Define a function $d(t)$ as

$$
d(t)=\left\{\begin{array}{ll}
t-t_{k} & t \in I_{1} \\
t-t_{k}-m h & t \in I_{2}^{m} \\
t-t_{k}-l h & t \in I_{3} .
\end{array}(m=1,2, \ldots, l-1)\right.
$$

From the definition of $d(t)$ defined in (2.12), we can derive

$$
\begin{gathered}
0 \leq d_{k} \leq d(t)<h+d, \quad t \in I_{1} \\
0 \leq d_{k} \leq d \leq d(t)<h+d, \quad t \in I_{2}^{m}(m=1,2, \ldots, l-1) \\
0 \leq d_{k} \leq d \leq d(t)<h+d, \quad t \in I_{3} .
\end{gathered}
$$

From (2.13), it can be derived that $0 \leq d(t)<d_{M}$, where $d_{M}=h+d$. For $t \in\left[t_{k}+d_{k}, t_{k+1}+d_{k+1}\right)$, we define

$$
e_{k}(t)=\left\{\begin{array}{ll}
0 & t \in I_{1} \\
x\left(t_{k}+m h\right)-x\left(t_{k}\right) & t \in I_{2}^{m} \\
x\left(t_{k}+l h\right)-x\left(t_{k}\right) & t \in I_{3}
\end{array}(m=1,2, \ldots, l-1)\right.
$$
as

Combining the above definitions of $d(t)$ and $e_{k}(t)$, the algorithm (2.5) can be rewritten

$$
e_{k}^{T}(t) C^{T} W C e_{k}(t)<\sigma x^{T}(t-d(t)) C^{T} W C x(t-d(t))
$$

Based on the available sampled measurement $\bar{y}(t)$, the following state estimator is adopted:

$$
\dot{\hat{x}}(t)=-A \widehat{x}(t)+K(\bar{y}(t)-C \widehat{x}(t))
$$

where $K$ is feedback gain matrix to be designed, and $\widehat{x}(t)=\left(\widehat{x}_{1}(t), \widehat{x}_{2}(t), \ldots, \widehat{x}_{n}(t)\right)^{T} \in \mathbb{R}^{n}$ is estimator state vector. 
Setting $e(t)=x(t)-\widehat{x}(t)$, the estimation error dynamics can be obtained from (2.1) and (2.16), and it follows that

$$
\dot{e}(t)=-(A+K C) e(t)+K C x(t)-K C x(t-d(t))+K C e_{k}(t)+W_{0} g(x(t))+W_{1} g(x(t-\tau(t))) .
$$

Let $\bar{x}(t)=\left(x^{T}(t), e^{T}(t)\right)^{T}$, we can get the following augmented system from (2.1) and

$$
\dot{\bar{x}}(t)=\bar{A} \bar{x}(t)+\bar{B} \bar{x}(t-d(t))+\bar{W}_{0} g(H \bar{x}(t))+\bar{W}_{1} g(H \bar{x}(t-\tau(t)))+\bar{C} e_{k}(t),
$$

where

$$
\begin{gathered}
\bar{A}=\left[\begin{array}{cc}
-A & 0 \\
K C & -A-K C
\end{array}\right] \quad \bar{B}=\left[\begin{array}{cc}
0 & 0 \\
-K C & 0
\end{array}\right] \quad \bar{W}_{0}=\left[\begin{array}{l}
W_{0} \\
W_{0}
\end{array}\right] \\
\bar{W}_{1}=\left[\begin{array}{l}
W_{1} \\
W_{1}
\end{array}\right] \quad H^{T}=\left[\begin{array}{l}
I \\
0
\end{array}\right] \quad \bar{C}=\left[\begin{array}{c}
0 \\
K C
\end{array}\right] .
\end{gathered}
$$

Before giving the main results, the following assumption, definition, and lemma are essential in establishing our main results.

Assumption 2.3 (see, [27]). The activation function $g(\cdot)$ satisfies the following sector-bounded condition:

$$
\left[g(x)-U_{1} x\right]^{T}\left[g(x)-U_{2} x\right] \leq 0
$$

where $U_{1}$ and $U_{2}$ are two real constant matrices with $U_{2}-U_{1} \geq 0$.

Definition 2.4 (see, [27]). The augmented system (2.18) is exponentially stable, if there exist two constants $\alpha>0$ and $\beta>0$, such that

$$
\|\bar{x}(t)\|^{2} \leq \alpha e^{-\beta t} \sup _{-r \leq \theta \leq 0}\|\phi(\theta)\|^{2}
$$

where $\phi(\cdot)$ is in the initial function system $(2.18)$ as $\phi(t)=\bar{x}(t), t \in[-r, 0]$. 
Lemma 2.5 (see, $[38,39])$. Suppose $\tau(t) \in\left[\tau_{m}, \tau_{M}\right], Q_{i}(i=1,2,3)$ are some constant matrices with appropriate dimensions, then

$$
Q_{1}+\left(\tau_{M}-\tau(t)\right) Q_{2}+\left(\tau(t)-\tau_{m}\right) Q_{3}<0
$$

if the following inequalities hold

$$
\begin{aligned}
& Q_{1}+\left(\tau_{M}-\tau_{m}\right) Q_{2}<0 \\
& Q_{1}+\left(\tau_{M}-\tau_{m}\right) Q_{3}<0 .
\end{aligned}
$$

Lemma 2.6 (see, [40]). For any constant positive matrix $T \in \mathbb{R}^{n \times n}$, scalar $\tau_{1} \leq \tau(t)<\tau_{2}$ and vector function $\dot{x}(t):\left[-\tau_{2}, \tau_{1}\right] \rightarrow \mathbb{R}^{n}$ such that the following integration is well defined, then it holds that

$$
-\left(\tau_{2}-\tau_{1}\right) \int_{t-\tau_{2}}^{t-\tau_{1}} \dot{x}^{T}(v) T \dot{x}(v) d v \leq\left[\begin{array}{c}
x\left(t-\tau_{1}\right) \\
x(t-\tau(t)) \\
x\left(t-\tau_{2}\right)
\end{array}\right]^{T}\left[\begin{array}{ccc}
-T & T & 0 \\
* & -2 T & T \\
* & * & -T
\end{array}\right]\left[\begin{array}{c}
x\left(t-\tau_{1}\right) \\
x(t-\tau(t)) \\
x\left(t-\tau_{2}\right)
\end{array}\right] .
$$

\section{Main Results}

In this section, we design a sampled-date estimator with form (2.18) for recurrent neural networks with time-varying delay based event-triggered control.

The system (2.18) can be rewritten as

$$
\dot{\bar{x}}(t)=\mathcal{A} \xi(t),
$$

where

$$
\begin{gathered}
\xi(t)=\left[\bar{x}^{T}(t), \bar{x}^{T}(t-d(t)), \bar{x}^{T}\left(t-d_{M}\right), \bar{x}^{T}(t-\tau(t)), \bar{x}^{T}(t-\tau), g^{T}(H \bar{x}(t)), g^{T}(H \bar{x}(t-\tau(t))), e_{k}^{T}(t)\right]^{T} \\
\mathcal{A}=\left[\bar{A}, \bar{B}, 0,0,0, \bar{W}_{0}, \bar{W}_{1}, \bar{C}\right] .
\end{gathered}
$$

Theorem 3.1. Suppose that Assumption 2.3 holds, for given estimator gain matrix $K$, the augmented system (3.1) is exponentially stable, if there exist some positive definite matrices $P>0, Q_{i}>0, R_{i}>0$ and $S_{i}, T_{i}(i=1,2)$ with appropriate dimension, and two positive scalars $\alpha>0, \beta>0$, such that the following linear matrix inequalities hold:

$$
\Pi_{i}=\left[\begin{array}{cccc}
\Pi & \Phi_{1} & \Phi_{2} & \Phi_{3}^{(i)} \\
* & -d_{M} R_{1} & 0 & 0 \\
* & * & -\tau R_{2} & 0 \\
* & * & * & -\tau R_{2}
\end{array}\right]<0 \quad(i=1,2)
$$


where

$$
\begin{aligned}
& \Pi=\left[\begin{array}{cccccccc}
\Pi_{11} & \Pi_{12} & 0 & \Pi_{13} & 0 & \Pi_{14} & P \bar{W}_{1} & P \bar{C} \\
* & \Pi_{22} & \frac{1}{d_{M}} R_{1} & 0 & 0 & 0 & 0 & 0 \\
* & * & \Pi_{33} & 0 & 0 & 0 & 0 & 0 \\
* & * & * & \Pi_{44} & \Pi_{45} & 0 & -\beta \bar{U}_{2} & 0 \\
* & * & * & * & \Pi_{55} & 0 & 0 & 0 \\
* & * & * & * & * & -\alpha I & 0 & 0 \\
* & * & * & * & * & * & -\beta I & 0 \\
* & * & * & * & * & * & * & -C^{T} W C
\end{array}\right] \\
& \Phi_{1}=\left[\begin{array}{llllllll}
d_{M} R_{1} \bar{A} & d_{M} R_{1} \bar{B} & 0 & 0 & 0 & d_{M} R_{1} \bar{W}_{0} & d_{M} R_{1} \bar{W}_{1} & d_{M} R_{1} \bar{C}
\end{array}\right]^{T} \\
& \Phi_{2}=\left[\begin{array}{llllllllll}
\tau R_{2} \bar{A} & \tau R_{2} \bar{B} & 0 & 0 & 0 & \tau R_{2} \bar{W}_{0} & \tau R_{2} \bar{W}_{1} & \tau R_{2} \bar{C}
\end{array}\right]^{T} \\
& \Phi_{3}^{(1)}=\left[\begin{array}{llllllll}
\tau S_{1}^{T} & 0 & 0 & \tau S_{2}^{T} & 0 & 0 & 0 & 0
\end{array}\right]^{T} \\
& \Phi_{3}^{(2)}=\left[\begin{array}{llllllll}
0 & 0 & 0 & \tau T_{1}^{T} & \tau T_{2}^{T} & 0 & 0 & 0
\end{array}\right]^{T} \\
& \Pi_{11}=P \bar{A}+\bar{A}^{T} P+Q_{1}+Q_{2}-\frac{1}{d_{M}} R_{1}+S_{1}+S_{1}^{T}-\alpha \bar{U}_{1} \\
& \Pi_{12}=P \bar{B}+\frac{1}{d_{M}} R_{1} \\
& \Pi_{13}=S_{2}^{T}-S_{1} \\
& \Pi_{14}=P \bar{W}_{0}-\alpha \bar{U}_{2} \\
& \Pi_{22}=-\frac{2}{d_{M}} R_{1}+\sigma \Omega \\
& \Pi_{33}=-Q_{1}-\frac{1}{d_{M}} R_{1} \\
& \Pi_{44}=-S_{2}-S_{2}^{T}+T_{1}+T_{1}^{T}-\beta \bar{U}_{1} \\
& \Pi_{45}=-T_{1}+T_{2}^{T} \\
& \Pi_{55}=-Q_{2}-T_{2}-T_{2}^{T} \\
& \Omega=\left[\begin{array}{cc}
C^{T} W C & 0 \\
0 & 0
\end{array}\right] .
\end{aligned}
$$

Proof. Construct the following Lyapunov-Krasovskii functional candidate:

$$
V(t, \bar{x}(t))=V_{1}(t, \bar{x}(t))+V_{2}(t, \bar{x}(t))+V_{3}(t, \bar{x}(t))+V_{4}(t, \bar{x}(t))
$$


where

$$
\begin{gathered}
V_{1}(t, \bar{x}(t))=\bar{x}^{T}(t) P \bar{x}(t) \\
V_{2}(t, \bar{x}(t))=\int_{t-d_{M}}^{t} \bar{x}^{T}(s) Q_{1} \bar{x}(s) d s+\int_{t-\tau}^{t} \bar{x}^{T}(s) Q_{2} \bar{x}(s) d s \\
V_{3}(t, \bar{x}(t))=\int_{t-d_{M}}^{t} \int_{\theta}^{t} \dot{\bar{x}}^{T}(s) R_{1} \dot{\bar{x}}(s) d s d \theta \\
V_{4}(t, \bar{x}(t))=\int_{t-\tau}^{t} \int_{\theta}^{t} \dot{\bar{x}}^{T}(s) R_{2} \dot{\bar{x}}(s) d s d \theta,
\end{gathered}
$$

and $P>0, Q_{i}>0$ and $R_{i}>0(i=1,2)$ are matrices to be determined.

The derivative of $V_{i}(t, \bar{x}(t))(i=1,2,3,4)$ along the trajectory of system (3.1) can be shown as follows:

$$
\begin{gathered}
\dot{V}_{1}(t, \bar{x}(t))=2 \bar{x}^{T}(t) P A \xi(t) \\
\dot{V}_{2}(t, \bar{x}(t))=\bar{x}^{T}(t)\left(Q_{1}+Q_{2}\right) \bar{x}(t)-\bar{x}^{T}\left(t-d_{M}\right) Q_{1} \bar{x}\left(t-d_{M}\right)-\bar{x}^{T}(t-\tau) Q_{2} \bar{x}(t-\tau) \\
\dot{V}_{3}(t, \bar{x}(t))=d_{M} \dot{\bar{x}}^{T}(t) R_{1} \dot{\bar{x}}(t)-\int_{t-d_{M}}^{t} \dot{\bar{x}}^{T}(s) R_{1} \dot{\bar{x}}(s) d s \\
=d_{M} \xi^{T}(t) A^{T} R_{1} A \xi(t)-\int_{t-d_{M}}^{t} \dot{\bar{x}}^{T}(s) R_{1} \dot{\bar{x}}(s) d s \\
\dot{V}_{4}(t, \bar{x}(t))=\tau \dot{\bar{x}}^{T}(t) R_{2} \dot{\bar{x}}(t)-\int_{t-\tau}^{t} \dot{\bar{x}}^{T}(s) R_{2} \dot{\bar{x}}(s) d s \\
=\tau \xi^{T}(t) \mathscr{A}^{T} R_{2} \mathscr{A} \xi(t)-\int_{t-\tau}^{t} \dot{\bar{x}}^{T}(s) R_{2} \dot{\bar{x}}(s) d s .
\end{gathered}
$$

Noting that (3.9), it follows from Lemma 2.6 that

$$
-\int_{t-d_{M}}^{t} \dot{\bar{x}}^{T}(s) R_{1} \dot{\bar{x}}(s) d s \leq \frac{1}{d_{M}}\left[\begin{array}{c}
\bar{x}(t) \\
\bar{x}(t-d(t)) \\
\bar{x}\left(t-d_{M}\right)
\end{array}\right]^{T}\left[\begin{array}{ccc}
-R_{1} & R_{1} & 0 \\
* & -2 R_{1} & R_{1} \\
* & * & -R_{1}
\end{array}\right]\left[\begin{array}{c}
\bar{x}(t) \\
\bar{x}(t-d(t)) \\
\bar{x}\left(t-d_{M}\right)
\end{array}\right] .
$$

Employing the free matrix method $[38,39]$, it is easily derived that

$$
\begin{gathered}
2 \xi^{T}(t) S\left[\bar{x}(t)-\bar{x}(t-\tau(t))-\int_{t-\tau(t)}^{t} \dot{\bar{x}}(s) d s\right]=0, \\
2 \xi^{T}(t) T\left[\bar{x}(t-\tau(t))-\bar{x}(t-\tau)-\int_{t-\tau}^{t-\tau(t)} \dot{\bar{x}}(s) d s\right]=0,
\end{gathered}
$$


where

$$
\begin{aligned}
& S=\left[\begin{array}{llllllll}
S_{1}^{T} & 0 & 0 & S_{2}^{T} & 0 & 0 & 0 & 0
\end{array}\right]^{T}, \\
& T=\left[\begin{array}{llllllll}
0 & 0 & 0 & T_{1}^{T} & T_{2}^{T} & 0 & 0 & 0
\end{array}\right]^{T} .
\end{aligned}
$$

It follows that from (3.12) that

$$
\begin{gathered}
-2 \xi^{T}(t) S \int_{t-\tau(t)}^{t} \dot{\bar{x}}(s) d s \leq \tau(t) \xi^{T}(t) S R_{2}^{-1} S^{T} \xi(t)+\int_{t-\tau(t)}^{t} \dot{\bar{x}}^{T}(s) R_{2} \dot{\bar{x}}(s) d s \\
-2 \xi^{T}(t) T \int_{t-\tau}^{t-\tau(t)} \dot{\bar{x}}(s) d s \leq(\tau-\tau(t)) \xi^{T}(t) T R_{2}^{-1} T^{T} \xi(t)+\int_{t-\tau}^{t-\tau(t)} \dot{\bar{x}}^{T}(s) R_{2} \dot{\bar{x}}(s) d s .
\end{gathered}
$$

By Assumption 2.3, the following inequality holds:

$$
\left[\begin{array}{c}
\bar{x}(t) \\
g(H \bar{x}(t))
\end{array}\right]^{T}\left[\begin{array}{cc}
\bar{U}_{1} & \bar{U}_{2} \\
\bar{U}_{2}^{T} & I
\end{array}\right]\left[\begin{array}{c}
\bar{x}(t) \\
g(H \bar{x}(t))
\end{array}\right] \leq 0
$$

where

$$
\begin{gathered}
\bar{U}_{1}=H^{T} \widehat{U}_{1} H, \quad \bar{U}_{2}=H^{T} \widehat{U}_{2} \\
\widehat{U}_{1}=\frac{U_{1}^{T} U_{2}+U_{2}^{T} U_{1}}{2}, \quad \widehat{U}_{2}=\frac{U_{1}^{T}+U_{2}^{T}}{2} .
\end{gathered}
$$

For all $\alpha, \beta>0$, it can be derived from (3.15) that

$$
\begin{gathered}
-\alpha\left[\begin{array}{c}
\bar{x}(t) \\
g(H \bar{x}(t))
\end{array}\right]^{T}\left[\begin{array}{cc}
\bar{U}_{1} & \bar{U}_{2} \\
\bar{U}_{2}^{T} & I
\end{array}\right]\left[\begin{array}{c}
\bar{x}(t) \\
g(H \bar{x}(t))
\end{array}\right] \geq 0 \\
-\beta\left[\begin{array}{c}
\bar{x}(t-\tau(t)) \\
g(H \bar{x}(t-\tau(t)))
\end{array}\right]^{T}\left[\begin{array}{cc}
\bar{U}_{1} & \bar{U}_{2} \\
\bar{U}_{2}^{T} & I
\end{array}\right]\left[\begin{array}{c}
\bar{x}(t-\tau(t)) \\
g(H \bar{x}(t-\tau(t)))
\end{array}\right] \geq 0 .
\end{gathered}
$$

Then, (2.15) can be rewritten as

$$
\sigma \bar{x}^{T}(t-d(t)) \Omega \bar{x}(t-d(t))-\left[\begin{array}{cc}
e_{k}^{T}(t) C^{T} W C e_{k}(t) & 0 \\
0 & 0
\end{array}\right]>0
$$

where

$$
\Omega=\left[\begin{array}{cc}
C^{T} W C & 0 \\
0 & 0
\end{array}\right]
$$


It follows from (3.7)-(3.18) that

$$
\begin{aligned}
\dot{V}(t, \bar{x}(t)) \leq & 2 \bar{x}^{T}(t) P \mathcal{A} \xi(t)+\bar{x}^{T}(t)\left(Q_{1}+Q_{2}\right) \bar{x}(t)-\bar{x}^{T}\left(t-d_{M}\right) Q_{1} \bar{x}\left(t-d_{M}\right) \\
& -\bar{x}^{T}(t-\tau) Q_{2} \bar{x}(t-\tau)+d_{M} \xi^{T}(t) \mathcal{A}^{T} R_{1} \mathcal{A} \xi(t)+\tau \xi^{T}(t) \mathcal{A}^{T} R_{2} \mathcal{A} \xi(t) \\
& +2 \xi^{T}(t) S(\bar{x}(t)-\bar{x}(t-\tau(t)))+2 \xi^{T}(t) T(\bar{x}(t-\tau(t))-\bar{x}(t-\tau)) \\
& +\frac{1}{d_{M}}\left[\begin{array}{c}
\bar{x}(t) \\
\bar{x}(t-d(t)) \\
\bar{x}\left(t-d_{M}\right)
\end{array}\right]^{T}\left[\begin{array}{ccc}
-R_{1} & R_{1} & 0 \\
* & -2 R_{1} & R_{1} \\
* & * & -R_{1}
\end{array}\right]\left[\begin{array}{c}
\bar{x}(t) \\
\bar{x}(t-d(t)) \\
\bar{x}\left(t-d_{M}\right)
\end{array}\right] \\
& -\alpha\left[\begin{array}{c}
\bar{x}(t) \\
g(H \bar{x}(t))
\end{array}\right]^{T}\left[\begin{array}{cc}
\bar{U}_{1} & \bar{U}_{2} \\
\bar{U}_{2}^{T} & I
\end{array}\right]\left[\begin{array}{cc}
\bar{x}(t) \\
g(H \bar{x}(t))
\end{array}\right] \\
& -\beta\left[\begin{array}{c}
\bar{x}(t-\tau(t)) \\
g(H \bar{x}(t-\tau(t)))
\end{array}\right]^{T}\left[\begin{array}{cc}
\bar{U}_{1} & \bar{U}_{2} \\
\bar{U}_{2}^{T} & I
\end{array}\right]\left[\begin{array}{c}
\bar{x}(t-\tau(t)) \\
g(H \bar{x}(t-\tau(t)))
\end{array}\right] \\
& +\sigma \bar{x}^{T}(t-d(t)) \Omega \bar{x}(t-d(t))-\left[\begin{array}{cc}
e_{k}^{T}(t) C^{T} W C e_{k}(t) & 0 \\
0 & 0
\end{array}\right] \\
& +\tau(t) \xi^{T}(t) S R_{2}^{-1} S^{T} \xi(t)+(\tau-\tau(t)) \xi^{T}(t) T R_{2}^{-1} T^{T} \xi(t) \\
= & \xi^{T}(t)\left(\Pi+d_{M} \mathscr{A}^{T} R_{1} \mathscr{A}+\tau \mathscr{A}^{T} R_{2} \mathscr{A}\right) \xi(t)+\tau(t) \xi^{T}(t) S R_{2}^{-1} S^{T} \xi(t) \\
& +(\tau-\tau(t)) \xi^{T}(t) T R_{2}^{-1} T^{T} \xi(t) .
\end{aligned}
$$

By using Schur complement and Lemma 2.5, it can be seen that (3.3) is equivalent to

$$
\Pi+d_{M} \mathscr{A}^{T} R_{1} \mathscr{A}+\tau \mathscr{A}^{T} R_{2} \mathscr{A}+\tau(t) S R_{2}^{-1} S^{T}+(\tau-\tau(t)) T R_{2}^{-1} T^{T}<0
$$

which implies $\dot{V}(t, \bar{x}(t))<-\varepsilon\|\bar{x}(t)\|^{2}$; then similar to [41], we can obtain the exponential stability of system (3.1). The proof is completed.

Remark 3.2. From Theorem 3.1, it can be seen that the trigger parameters $\sigma, W$ and the upper bound of time delay $\tau$ are involved in (3.3); for given $\sigma$, the corresponding trigger parameter $W$ and the upper bound of $\tau$ can be obtained by using LMI toolbox in Matlab. From the simulation example, it can be derived that the larger the $\sigma$, the small the $\tau$; the larger average release period, which means the load of network transmission will be reduced.

Remark 3.3. When the estimator gain matrix $K$ is given, the conditions (3.3) are in the form of linear matrix inequalities, which can be readily solved by using the standard numerical software. The conditions (3.3) are not linear matrix inequalities when the estimator gain matrix $K$ is a matrix variable to be designed, and thus Theorem 3.1 cannot be used to design $K$ directly, a design method will be provided in the following Theorem. 
After establishing analysis results in Theorem 3.1, the design problem of state estimator is to be considered and the following results can be readily derived from Theorem 3.1.

Theorem 3.4. Suppose that Assumption 2.3 holds, the augmented system (3.1) is exponentially stable, if there exist $P=\operatorname{diag}\left\{\mathbf{P}_{1}, \mathbf{P}_{2}\right\}>0, Q_{i}=\operatorname{diag}\left\{\mathbf{Q}_{i}, \mathbf{Q}_{i}\right\}>0, R_{i}=\operatorname{diag}\left\{\mathbf{R}_{i}, \mathbf{R}_{i}\right\}>0$ and $S_{i}=\operatorname{diag}\left\{\mathbf{S}_{i}, \mathbf{S}_{i}\right\}, T_{i}=\operatorname{diag}\left\{\mathbf{T}_{i}, \mathbf{T}_{i}\right\}(i=1,2)$ and $V$ with appropriate dimension, and two positive scalars $\alpha>0, \beta>0$, such that the following linear matrix inequalities hold:

$$
\bar{\Pi}_{i}=\left[\begin{array}{cccc}
\bar{\Pi} & \bar{\Phi}_{1} & \bar{\Phi}_{2} & \bar{\Phi}_{3}^{(i)} \\
* & \bar{\Phi}_{4} & 0 & 0 \\
* & * & \bar{\Phi}_{5} & 0 \\
* & * & * & -\tau R_{2}
\end{array}\right]<0 \quad(i=1,2)
$$

where

$$
\begin{aligned}
& \bar{\Pi}=\left[\begin{array}{cccccccc}
\bar{\Pi}_{11} & \bar{\Pi}_{12} & 0 & \bar{\Pi}_{13} & 0 & \bar{\Pi}_{14} & \bar{\Pi}_{15} & \bar{\Pi}_{16} \\
* & \bar{\Pi}_{22} & \bar{\Pi}_{23} & 0 & 0 & 0 & 0 & 0 \\
* & * & \bar{\Pi}_{33} & 0 & 0 & 0 & 0 & 0 \\
* & * & * & \bar{\Pi}_{44} & \bar{\Pi}_{45} & 0 & \bar{\Pi}_{46} & 0 \\
* & * & * & * & \bar{\Pi}_{55} & 0 & 0 & 0 \\
* & * & * & * & * & \bar{\Pi}_{66} & 0 & 0 \\
* & * & * & * & * & * & \bar{\Pi}_{77} & 0 \\
* & * & * & * & * & * & * & \bar{\Pi}_{88}
\end{array}\right] \\
& \bar{\Phi}_{1}=\left[\begin{array}{llllllll}
\bar{\Pi}_{17}^{T} & \bar{\Pi}_{24}^{T} & 0 & 0 & 0 & \bar{\Pi}_{67}^{T} & \bar{\Pi}_{78}^{T} & \bar{\Pi}_{89}^{T}
\end{array}\right]^{T}
\end{aligned}
$$

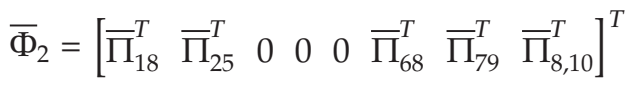

$$
\begin{aligned}
& \bar{\Phi}_{3}^{(1)}=\left[\begin{array}{llllllll}
\bar{\Pi}_{19}^{T} & 0 & 0 & \bar{\Pi}_{47}^{T} & 0 & 0 & 0 & 0
\end{array}\right]^{T} \\
& \Phi_{3}^{(2)}=\left[\begin{array}{llllllll}
0 & 0 & 0 & \widehat{\Pi}_{19}^{T} & \widehat{\Pi}_{47}^{T} & 0 & 0 & 0
\end{array}\right]^{T} \\
& \bar{\Phi}_{4}=\left[\begin{array}{cc}
2 d_{M} \mathbf{P}_{1}+d_{M} \mathbf{R}_{1} & 0 \\
0 & 2 d_{M} \mathbf{P}_{2}+d_{M} \mathbf{R}_{1}
\end{array}\right] \\
& \bar{\Phi}_{5}=\left[\begin{array}{cc}
2 \tau \mathbf{P}_{1}+\tau \mathbf{R}_{2} & 0 \\
0 & 2 \tau \mathbf{P}_{2}+\tau \mathbf{R}_{2}
\end{array}\right] \\
& \bar{\Pi}_{11}=\left[\begin{array}{cc}
-\mathbf{P}_{1} A-A^{T} \mathbf{P}_{1}-\alpha \widehat{U}_{1} & C^{T} V^{T} \\
V C & -\mathbf{P}_{2} A-A^{T} \mathbf{P}_{2}-V C-C^{T} V^{T}
\end{array}\right] \\
& +\left[\begin{array}{cc}
\mathbf{Q}_{1}+\mathbf{Q}_{2}-\frac{1}{d_{M}} \mathbf{R}_{1}+\mathbf{S}_{1}+\mathbf{S}_{1}^{T} & 0 \\
0 & \mathbf{Q}_{1}+\mathbf{Q}_{2}-\frac{1}{d_{M}} \mathbf{R}_{1}+\mathbf{S}_{1}+\mathbf{S}_{1}^{T}
\end{array}\right]
\end{aligned}
$$




$$
\begin{aligned}
& \bar{\Pi}_{12}=\left[\begin{array}{cc}
\frac{1}{d_{M}} \mathbf{R}_{1} & 0 \\
V C & \frac{1}{d_{M}} \mathbf{R}_{1}
\end{array}\right], \quad \bar{\Pi}_{13}=\left[\begin{array}{cc}
\mathbf{S}_{2}^{T}-\mathbf{S}_{1} & 0 \\
0 & \mathbf{S}_{2}^{T}-\mathbf{S}_{1}
\end{array}\right] \\
& \bar{\Pi}_{14}=\left[\begin{array}{c}
\mathbf{P}_{1} W_{0}-\alpha \widehat{U}_{2} \\
\mathbf{P}_{2} W_{0}
\end{array}\right], \quad \bar{\Pi}_{15}=\left[\begin{array}{c}
\mathbf{P}_{1} W_{1} \\
\mathbf{P}_{2} W_{1}
\end{array}\right] \\
& \bar{\Pi}_{16}=\left[\begin{array}{c}
0 \\
V C
\end{array}\right], \quad \bar{\Pi}_{17}=\left[\begin{array}{cc}
-d_{M} A^{T} \mathbf{P}_{1} & d_{M} C^{T} V^{T} \\
0 & -d_{M} A^{T} \mathbf{P}_{2}-d_{M} C^{T} V^{T}
\end{array}\right] \\
& \bar{\Pi}_{18}=\left[\begin{array}{cc}
-\tau A^{T} \mathbf{P}_{1} & \tau C^{T} V^{T} \\
0 & -\tau A^{T} \mathbf{P}_{2}-\tau C^{T} V^{T}
\end{array}\right], \quad \bar{\Pi}_{19}=\left[\begin{array}{cc}
\tau \mathbf{S}_{1} & 0 \\
0 & \tau \mathbf{S}_{1}
\end{array}\right] \\
& \widehat{\Pi}_{19}=\left[\begin{array}{cc}
\tau \mathbf{T}_{1} & 0 \\
0 & \tau \mathbf{T}_{1}
\end{array}\right], \quad \bar{\Pi}_{22}=\left[\begin{array}{cc}
-\frac{2}{d_{M}} \mathbf{R}_{1}+\sigma C^{T} W C & 0 \\
0 & -\frac{2}{d_{M}} \mathbf{R}_{1}
\end{array}\right] \\
& \bar{\Pi}_{23}=\left[\begin{array}{cc}
\frac{1}{d_{M}} \mathbf{R}_{1} & 0 \\
0 & \frac{1}{d_{M}} \mathbf{R}_{1}
\end{array}\right], \quad \bar{\Pi}_{24}=\left[\begin{array}{cc}
0 & -d_{M} C^{T} V^{T} \\
0 & 0
\end{array}\right] \\
& \bar{\Pi}_{25}=\left[\begin{array}{cc}
0 & \tau C^{T} V^{T} \\
0 & 0
\end{array}\right], \quad \bar{\Pi}_{33}=\left[\begin{array}{cc}
-\mathbf{Q}_{1}-\frac{1}{d_{M}} \mathbf{R}_{1} & 0 \\
0 & -\mathbf{Q}_{1}-\frac{1}{d_{M}} \mathbf{R}_{1}
\end{array}\right] \\
& \bar{\Pi}_{44}=\left[\begin{array}{cc}
-\mathbf{S}_{2}-\mathbf{S}_{2}^{T}+\mathbf{T}_{1}+\mathbf{T}_{1}^{T}-\beta \widehat{U}_{1} & 0 \\
0 & -\mathbf{S}_{2}-\mathbf{S}_{2}^{T}+\mathbf{T}_{1}+\mathbf{T}_{1}^{T}
\end{array}\right], \quad \bar{\Pi}_{45}=\left[\begin{array}{cc}
\mathbf{T}_{2}^{T}-\mathbf{T}_{1} & 0 \\
0 & \mathbf{T}_{2}^{T}-\mathbf{T}_{1}
\end{array}\right] \\
& \bar{\Pi}_{46}=\left[\begin{array}{c}
-\beta \widehat{U}_{2} \\
0
\end{array}\right], \quad \bar{\Pi}_{47}=\left[\begin{array}{cc}
\tau \mathbf{S}_{2} & 0 \\
0 & \tau \mathbf{S}_{2}
\end{array}\right], \quad \widehat{\Pi}_{47}=\left[\begin{array}{cc}
\tau \mathbf{T}_{2} & 0 \\
0 & \tau \mathbf{T}_{2}
\end{array}\right] \\
& \bar{\Pi}_{55}=\left[\begin{array}{cc}
-\mathbf{Q}_{2}-\mathbf{T}_{2}-\mathbf{T}_{2}^{T} & 0 \\
0 & -\mathbf{Q}_{2}-\mathbf{T}_{2}-\mathbf{T}_{2}^{T}
\end{array}\right], \quad \bar{\Pi}_{66}=-\alpha I \\
& \bar{\Pi}_{67}=\left[\begin{array}{lll}
d_{M} W_{0}^{T} \mathbf{P}_{1} & d_{M} W_{0}^{T} \mathbf{P}_{2}
\end{array}\right], \quad \bar{\Pi}_{68}=\left[\begin{array}{lll}
\tau W_{0}^{T} \mathbf{P}_{1} & \tau W_{0}^{T} \mathbf{P}_{2}
\end{array}\right], \quad \bar{\Pi}_{77}=-\beta I \\
& \bar{\Pi}_{78}=\left[\begin{array}{lll}
d_{M} W_{1}^{T} \mathbf{P}_{1} & d_{M} W_{1}^{T} \mathbf{P}_{2}
\end{array}\right], \quad \bar{\Pi}_{79}=\left[\begin{array}{lll}
\tau W_{1}^{T} \mathbf{P}_{1} & \tau W_{1}^{T} \mathbf{P}_{2}
\end{array}\right], \quad \bar{\Pi}_{88}=-C^{T} W C \\
& \bar{\Pi}_{89}=\left[\begin{array}{ll}
0 & -d_{M} C^{T} V^{T}
\end{array}\right], \quad \bar{\Pi}_{8,10}=\left[\begin{array}{ll}
0 & -\tau C^{T} V^{T}
\end{array}\right],
\end{aligned}
$$

then the desired estimator gain matrix is given as $\mathrm{K}=\mathbf{P}_{2}^{-1} \mathrm{~V}$.

Proof. By using Schur complement in Theorem 3.1, $\Pi_{i}<0(i=1,2)$ can be rewritten as

$$
\begin{aligned}
& \Pi+d_{M} \mathscr{A}^{T} R_{1} \mathscr{A}+\tau \mathscr{A}^{T} R_{2} \mathscr{A}+\tau S R_{2}^{-1} S^{T}<0 \\
& \Pi+d_{M} \mathscr{A}^{T} R_{1} \mathscr{A}+\tau \mathscr{A}^{T} R_{2} \mathscr{A}+\tau T R_{2}^{-1} T^{T}<0
\end{aligned}
$$


By using Lemma 2.5, (3.25) are equivalent to the following matrix inequalities

$$
\left[\begin{array}{cccc}
\bar{\Pi} & \widehat{\Phi}_{1} & \widehat{\Phi}_{2} & \Phi_{3}^{(i)} \\
* & -d_{M} R_{1}^{-1} & 0 & 0 \\
* & * & -\tau R_{2}^{-1} & 0 \\
* & * & * & -\tau R_{2}
\end{array}\right]<0 \quad(i=1,2)
$$

where

$$
\begin{gathered}
\bar{\Pi}=\left[\begin{array}{cccccccc}
\bar{\Pi}_{11} & \bar{\Pi}_{12} & 0 & \bar{\Pi}_{13} & 0 & \bar{\Pi}_{14} & \bar{\Pi}_{15} & \bar{\Pi}_{16} \\
* & \bar{\Pi}_{22} & \bar{\Pi}_{23} & 0 & 0 & 0 & 0 & 0 \\
* & * & \bar{\Pi}_{33} & 0 & 0 & 0 & 0 & 0 \\
* & * & * & \bar{\Pi}_{44} & \bar{\Pi}_{45} & 0 & \bar{\Pi}_{46} & 0 \\
* & * & * & * & \bar{\Pi}_{55} & 0 & 0 & 0 \\
* & * & * & * & * & \bar{\Pi}_{66} & 0 & 0 \\
* & * & * & * & * & * & \bar{\Pi}_{77} & 0 \\
* & * & * & * & * & * & * & \bar{\Pi}_{88}
\end{array}\right] \\
\widehat{\Phi}_{1}=\left[\begin{array}{cccccccc}
d_{M} \bar{A} & d_{M} \bar{B} & 0 & 0 & 0 & d_{M} \bar{W}_{0} & d_{M} \bar{W}_{1} & d_{M} \bar{C}
\end{array}\right]^{T} \\
\widehat{\Phi}_{2}=\left[\begin{array}{llllllll}
\tau \bar{A} & \tau \bar{B} & 0 & 0 & 0 & \tau \bar{W}_{0} & \tau \bar{W}_{1} & \tau \bar{C}
\end{array}\right]^{T} .
\end{gathered}
$$

Then performing a congruence transformation of $\operatorname{diag}\{I, P, P, I\}$ to (3.26), it can be derived that

$$
\left[\begin{array}{cccc}
\bar{\Pi} & \tilde{\Phi}_{1} & \widetilde{\Phi}_{2} & \Phi_{3}^{(i)} \\
* & -d_{M} P R_{1}^{-1} P & 0 & 0 \\
* & * & -\tau P R_{2}^{-1} P & 0 \\
* & * & * & -\tau R_{2}
\end{array}\right]<0 \quad(i=1,2)
$$

where

$$
\begin{aligned}
& \widetilde{\Phi}_{1}=\left[\begin{array}{llllllllll}
d_{M} P \bar{A} & d_{M} P \bar{B} & 0 & 0 & 0 & d_{M} P \bar{W}_{0} & d_{M} P \bar{W}_{1} & d_{M} P \bar{C}
\end{array}\right]^{T} \\
& \widetilde{\Phi}_{2}=\left[\begin{array}{lllllllll}
\tau P \bar{A} & \tau P \bar{B} & 0 & 0 & 0 & \tau P \bar{W}_{0} & \tau P \bar{W}_{1} & \tau P \bar{C}
\end{array}\right]^{T} .
\end{aligned}
$$

Setting $P_{2} K=V$ in (3.28) and considering the following inequality:

$$
-P R_{i}^{-1} P \leq-2 P+R_{i} \quad(i=1,2)
$$


By using (3.30), we can obtain

$$
\left[\begin{array}{cccc}
\bar{\Pi} & \widetilde{\Phi}_{1} & \widetilde{\Phi}_{2} & \Phi_{3}^{(i)} \\
* & -d_{M} P R_{1}^{-1} P & 0 & 0 \\
* & * & -\tau P R_{2}^{-1} P & 0 \\
* & * & * & -\tau R_{2}
\end{array}\right]<\left[\begin{array}{cccc}
\bar{\Pi} & \widetilde{\Phi}_{1} & \widetilde{\Phi}_{2} & \Phi_{3}^{(i)} \\
* & -2 d_{M} P+d_{M} R_{1} & 0 & 0 \\
* & * & -2 \tau P+\tau R_{2} & 0 \\
* & * & * & -\tau R_{2}
\end{array}\right] .
$$

Substitute $\bar{A}, \bar{B}, \bar{W}_{0}, H, \bar{W}_{1}, \bar{C}, P, Q_{i}, R_{i}, S_{i}, T_{i}(i=1,2)$ into the right of (3.31), combining (3.22), we can obtain

$$
\left[\begin{array}{cccc}
\bar{\Pi} & \tilde{\Phi}_{1} & \tilde{\Phi}_{2} & \Phi_{3}^{(i)} \\
* & -2 d_{M} P+d_{M} R_{1} & 0 & 0 \\
* & * & -2 \tau P+\tau R_{2} & 0 \\
* & * & * & -\tau R_{2}
\end{array}\right]<0 \quad(i=1,2)
$$

The rest of the proof follows directly from Theorem 3.1.

Remark 3.5. When the estimator gain matrix $K$ is a matrix variable to be designed, in order to transform the conditions (3.3) to linear matrix inequalities, and meanwhile reduce the computational complexity (i.e., reduce the number of matrix variables), in Theorem 3.4, matrix variables in Theorem 3.1 are replaced by some diagonal matrices. Then setting $P_{2} \mathrm{~K}=$ $V$, we can obtain (3.22), which is in the form of linear matrix inequalities, which are easy to be verified by LMI toolbox.

Remark 3.6. It is noticed that $d_{M}=h+d$, if $d_{M}$ is solved, we can select a sampling period $h<d_{M}$. For given $d$, the maximal allowable sampling period $h_{\max }$ can be obtained by the following two-step procedure.

(1) For given $\tau$ and $d$, setting $h_{\max }=h_{0}$ and step size STEP $=\operatorname{STEP}_{0}$, where $h_{0}$ and $\mathrm{STEP}_{0}$ are two specified positive constants.

(2) If LMIs (3.22) are feasible, set $h_{\max }=h_{0}+\mathrm{STEP}_{0}$ and return to step (2): otherwise, $h$ is the maximal allowable sampling period.

\section{Numerical Results}

In this section, a numerical example is given to verify the effectiveness of the proposed control techniques for estimation of recurrent neural networks with time-varying delays.

Example 4.1. Consider recurrent neural networks (2.1) with the following parameters

$$
A=\left[\begin{array}{cc}
1.5 & 0 \\
0 & 2
\end{array}\right], \quad W_{0}=\left[\begin{array}{cc}
0.3 & -0.4 \\
-0.4 & 0.3
\end{array}\right], \quad W_{1}=\left[\begin{array}{cc}
0.3 & 0.3 \\
0.3 & 0.3
\end{array}\right], \quad C=\left[\begin{array}{cc}
0.9 & 0.8 \\
0.7 & 0.5
\end{array}\right] .
$$

The neuron activation function is described as follows:

$$
g(x)=\left[\begin{array}{c}
0.5 x_{1}(t)-\tanh \left(0.2 x_{1}(t)\right)+0.2 x_{2}(t) \\
0.95 x_{2}(t)-\tanh \left(0.75 x_{2}(t)\right)
\end{array}\right] .
$$


Table 1: $d_{M}=0.01$.

\begin{tabular}{lccccccc}
\hline$\sigma$ & 0 & 0.01 & 0.1 & 0.2 & 0.3 & 0.4 & 0.5 \\
\hline$\tau$ & 1.2134 & 1.1966 & 1.1572 & 1.1570 & 1.1569 & 1.1569 & 1.1569 \\
\hline
\end{tabular}

Table 2: $\tau=1, d=0.01$.

\begin{tabular}{lccccccc}
\hline$\sigma$ & 0 & 0.01 & 0.1 & 0.15 & 0.2 & 0.3 & 0.99 \\
\hline$h_{\max }$ & 0.2244 & 0.2106 & 0.1998 & 0.1998 & 0.1998 & 0.1998 & 0.1998 \\
\hline
\end{tabular}

It is easy to verify that the nonlinear function $f(\cdot)$ satisfies Assumption 2.3; by some simple calculations, we can obtain

$$
U_{1}=\left[\begin{array}{cc}
0.3 & 0.2 \\
0 & 0.2
\end{array}\right] \quad U_{2}=\left[\begin{array}{cc}
0.5 & 0.2 \\
0 & 0.95
\end{array}\right]
$$

Setting $d_{M}=0.01$ and $\sigma=0.1$, by applying Theorem 3.4, it can be obtained the maximum allowable delay $\tau=1.1572$. More detailed calculation results for different values of $\sigma$ are given in Table 1. It can be shown that the larger $\sigma$, the smaller $\tau$. For given $\tau=1$ and $d=0.01$, based on Remark 3.6, we can obtain the maximal allowable sampling period $h_{\max }$, which are shown in Table 2. For given $\tau=1, \sigma=0.1$ and $d_{M}=0.01$, by using LMI Toolbox in LMIs (3.22), the feasible solution can be obtained as follows:

$$
\begin{array}{rlr}
\mathbf{P}_{1}=\left[\begin{array}{cc}
5.4676 & -0.1329 \\
-0.1329 & 5.4000
\end{array}\right], & \mathbf{P}_{2}=\left[\begin{array}{cc}
5.0204 & -0.1155 \\
-0.1155 & 4.2212
\end{array}\right], & \mathbf{Q}_{1}=\left[\begin{array}{cc}
2.7863 & -0.0955 \\
-0.0955 & 2.0982
\end{array}\right] \\
\mathbf{Q}_{2}=\left[\begin{array}{cc}
3.2539 & -0.0133 \\
-0.0133 & 2.8910
\end{array}\right], & \mathbf{R}_{1}=\left[\begin{array}{ll}
0.0222 & 0.0010 \\
0.0010 & 0.0234
\end{array}\right], & \mathbf{R}_{2}=\left[\begin{array}{cc}
2.2237 & -0.0246 \\
-0.0246 & 1.4752
\end{array}\right] \\
\mathbf{S}_{1}=\left[\begin{array}{cc}
-0.5503 & 0.0195 \\
0.0478 & -0.4156
\end{array}\right], & \mathbf{S}_{2}=\left[\begin{array}{ll}
1.0185 & 0.0674 \\
0.1378 & 1.1920
\end{array}\right], & \mathbf{T}_{1}=\left[\begin{array}{ll}
-1.0844 & -0.0634 \\
-0.0902 & -1.2378
\end{array}\right] \\
\mathbf{T}_{2}=\left[\begin{array}{cc}
0.3511 & -0.0221 \\
-0.0195 & 0.3384
\end{array}\right], & V=\left[\begin{array}{ll}
-0.0792 & -0.1282 \\
-0.1755 & -0.0863
\end{array}\right], & \alpha=6.4604, \beta=5.7723 .
\end{array}
$$

Then the triggered matrix and the desired estimator can be obtained as follows:

$$
W=\left[\begin{array}{cc}
4.6153 & -2.7354 \\
-2.7354 & 6.5131
\end{array}\right], \quad K=\left[\begin{array}{cc}
-0.0165 & -0.0308 \\
-0.0354 & -0.0214
\end{array}\right]
$$

For giving the sampling period $h=0.005$, Table 3 gives the relation of the trigger parameter $\sigma$, trigger times, the average release period, and the percentage of data transmissions; it can be seen that the larger the $\sigma$, the smaller trigger times; the larger average release period, the smaller percentage of data transmission, which are reasonable results. In the following, we provide some simulation results: when $\sigma=0$, the time varying delay $\tau(t)$ obeys uniform distribution over $[0,1]$, and the curves of the error dynamics of the neural networks $e_{i}(t)(i=1,2)$ are depicted in Figure 1, from which we can see the errors converge to zero asymptotically. If setting $\sigma=0.1$, The response of the error dynamics for the delayed 


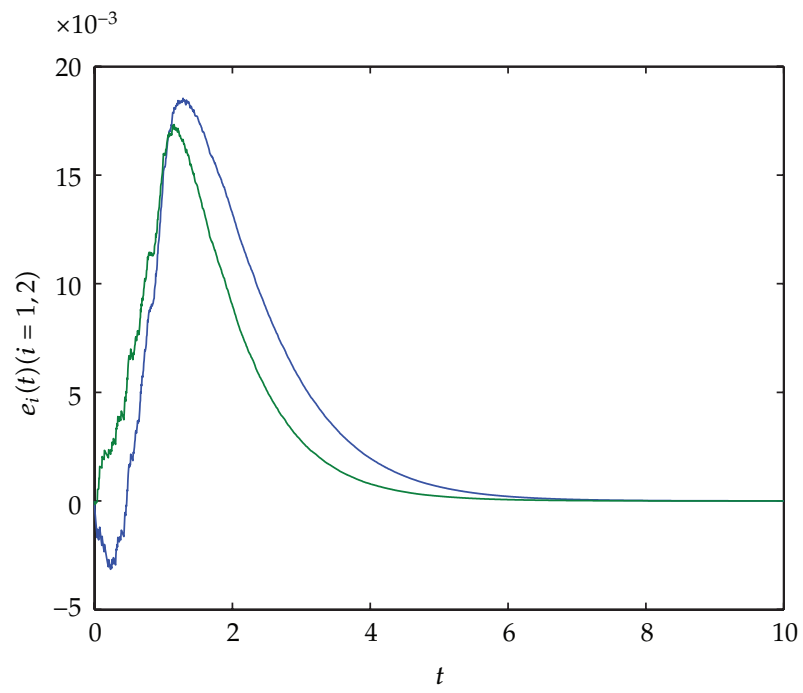

Figure 1: The error curves $e_{i}(t)(i=1,2)$ with trigger parameter $\sigma=0$ (time-triggered scheme).

Table 3: $h=0.005, d_{M}=0.01, \tau=0.1, t=10$.

\begin{tabular}{lccc}
\hline$\sigma$ & 0 & 0.01 & 0.1 \\
\hline Trigger times & 2000 & 188 & 74 \\
Trigger matrix $W$ & {$\left[\begin{array}{cc}0.7582 & -0.2843 \\
-0.2843 & 0.9490\end{array}\right]$} & {$\left[\begin{array}{cc}0.7504 & -0.2881 \\
-0.2881 & 0.9444\end{array}\right]$} & {$\left[\begin{array}{cc}4.6153 & -2.7354 \\
-2.7354 & 6.5131\end{array}\right]$} \\
Estimator matrix $K$ & {$\left[\begin{array}{cc}-0.0070 & -0.0553 \\
-0.0398 & -0.0257\end{array}\right]$} & {$\left[\begin{array}{cc}-0.0068 & -0.0552 \\
-0.0394 & -0.0255\end{array}\right]$} & {$\left[\begin{array}{cc}-0.0165 & -0.0308 \\
-0.0354 & -0.0214\end{array}\right]$} \\
Average release period & 0.0050 & 0.0531 & 0.1348 \\
Data transmission & $100 \%$ & $9.42 \%$ & $3.71 \%$ \\
\hline
\end{tabular}

neural networks (2.17) which converge to zero asymptotically in the mean square is given in Figure 2. Figure 3 shows the event-triggered release instants and intervals. It can be seen from Figures 1 and 2 that the simulation results are almost the same, but the percentage of data transmission under even-triggered scheme used much small number than time-triggering scheme. To make this clear, seen the computation results lists in Table 2, from which we can see that data transmission rate with even-triggered scheme $(\sigma=0.1)$ is only $3.71 \%$ of sampled measurement output with time-triggered scheme $(\sigma=0)$; from these results, we can draw a conclusion that event-triggered scheme has advantage over the time-triggered scheme in improving the resource utilization.

\section{Conclusions}

This paper has provided a novel event-triggered scheme to investigate the sampled-data state estimation problem for a class of recurrent neural networks with time-varying delays. This scheme can lead to a significant reduction of the information communication burden in the 


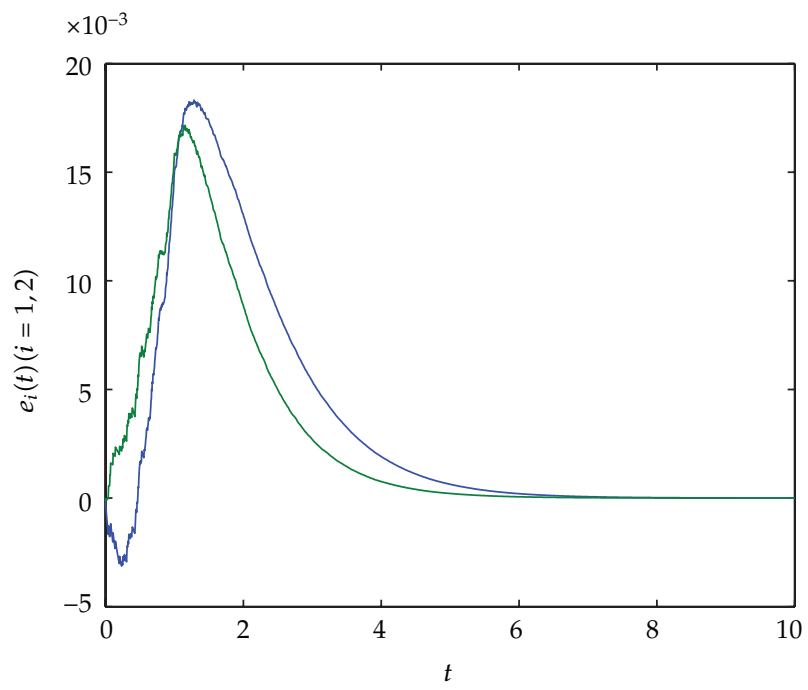

Figure 2: The error curves $e_{i}(t)(i=1,2)$ with trigger parameter $\sigma=0.1$ (event-triggered scheme).

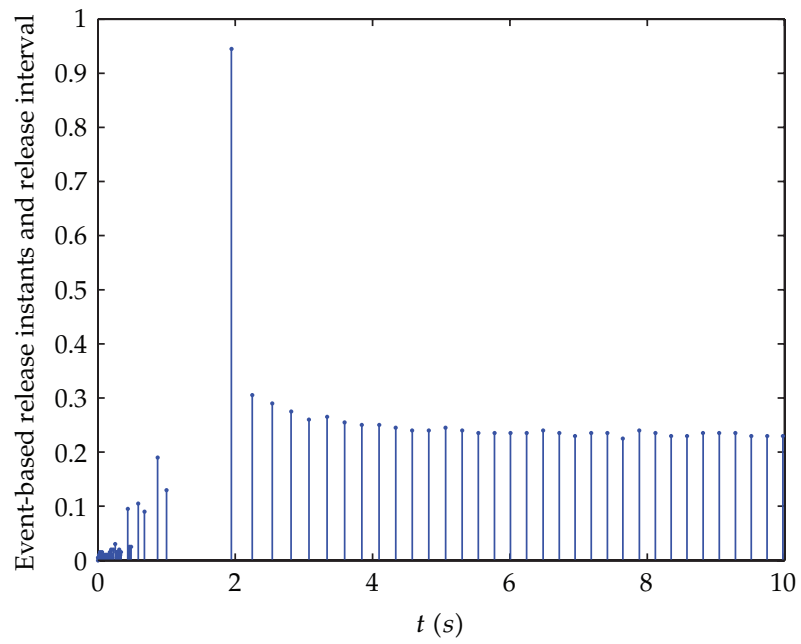

Figure 3: Release instants and release interval by event-triggered scheme.

network. By using a delayed-input approach, the error dynamics system is equivalently to a dynamic system with two different time-varying delays. Based on the Lyapunov-krasovskii functional approach, a state estimator of the considered neural networks can be achieved by solving some linear matrix inequalities, which can be readily solved by using the standard numerical software. Finally, an illustrative example is exploited to show the effectiveness of the event-triggered scheme. 


\section{Acknowledgments}

This work was in part jointly supported by the National Science Foundation of China (under Grant 61074024, 60874113), the Natural Science Foundation of Jiangsu Province of China (under Grant BK2010543), the Education Department Research Project of Zhejiang Province of China (under Grant Y201019013), and the Outstanding Young Teacher Project of Zhejiang Province.

\section{References}

[1] S. S. Young, P. D. Scott, and N. M. Nasrabadi, "Object recognition using multilayer Hopfield neural network," IEEE Transactions on Image Processing, vol. 6, no. 3, pp. 357-372, 1997.

[2] A. N. Michel, J. A. Farrell, and H.-F. Sun, "Analysis and synthesis techniques for Hopfield type synchronous discrete time neural networks with application to associative memory," Institute of Electrical and Electronics Engineers, vol. 37, no. 11, pp. 1356-1366, 1990.

[3] C. K. Ahn, "Robust stability of recurrent neural networks with ISS learning algorithm," Nonlinear Dynamics, vol. 65, no. 4, pp. 413-419, 2011.

[4] J. Liang and J. Cao, "Global output convergence of recurrent neural networks with distributed delays," Nonlinear Analysis: Real World Applications, vol. 8, no. 1, pp. 187-197, 2007.

[5] J. Cao and J. Wang, "Global asymptotic stability of a general class of recurrent neural networks with time-varying delays," IEEE Transactions on Circuits and Systems, vol. 50, no. 1, pp. 34-44, 2003.

[6] H. Zhang, Z. Liu, G. B. Huang, and Z. Wang, "Novel weighting-delay-based stability criteria for recurrent neural networks with time-varying delay," IEEE Transactions on Neural Networks, vol. 21, no. 1, pp. 91-106, 2010.

[7] J. Cao and L. Li, "Cluster synchronization in an array of hybrid coupled neural networks with delay," Neural Networks, vol. 22, no. 4, pp. 335-342, 2009.

[8] J. Cao, G. Chen, and P. Li, "Global synchronization in an array of delayed neural networks with hybrid coupling," IEEE Transactions on Systems, Man, and Cybernetics B, vol. 38, no. 2, pp. 488-498, 2008.

[9] P. Balasubramaniam, R. Krishnasamy, and R. Rakkiyappan, "Delay-dependent stability of neutral systems with time-varying delays using delay-decomposition approach," Applied Mathematical Modelling, vol. 36, no. 5, pp. 2253-2261, 2012.

[10] Z. Liu, H. Zhang, and Q. Zhang, "Novel stability analysis for recurrent neural networks with multiple delays via line integral-type L-K functional," IEEE Transactions on Neural Networks, vol. 21, no. 11, pp. 1710-1718, 2010.

[11] J. H. Park and O. M. Kwon, "Global stability for neural networks of neutral-type with interval timevarying delays," Chaos, Solitons and Fractals, vol. 41, no. 3, pp. 1174-1181, 2009.

[12] J. H. Park, "On global stability criterion of neural networks with continuously distributed delays," Chaos, Solitons and Fractals, vol. 37, no. 2, pp. 444-449, 2008.

[13] Z. Wang, D. W. C. Ho, and X. Liu, "State estimation for delayed neural networks," IEEE Transactions on Neural Networks, vol. 16, no. 1, pp. 279-284, 2005.

[14] J. H. Park and O. M. Kwon, "Design of state estimator for neural networks of neutral-type," Applied Mathematics and Computation, vol. 202, no. 1, pp. 360-369, 2008.

[15] J. H. Park and O. M. Kwon, "Further results on state estimation for neural networks of neutral-type with time-varying delay," Applied Mathematics and Computation, vol. 208, no. 1, pp. 69-75, 2009.

[16] Y. Liu, Z. Wang, and X. Liu, "State estimation for discrete-time Markovian jumping neural networks with mixed mode-dependent delays," Physics Letters A, vol. 372, no. 48, pp. 7147-7155, 2008.

[17] P. Balasubramaniam, S. Lakshmanan, and S. Jeeva Sathya Theesar, "State estimation for Markovian jumping recurrent neural networks with interval time-varying delays," Nonlinear Dynamics, vol. 60, no. 4, pp. 661-675, 2010.

[18] Z. Wang, Y. Liu, and X. Liu, "State estimation for jumping recurrent neural networks with discrete and distributed delays," Neural Networks, vol. 22, no. 1, pp. 41-48, 2009.

[19] Y. Liu, Z. Wang, J. Liang, and X. Liu, "Synchronization and state estimation for discrete-time complex networks with distributed delays," IEEE Transactions on Systems, Man, and Cybernetics B, vol. 38, no. 5, pp. 1314-1325, 2008. 
[20] B. Shen, Z. Wang, and X. Liu, "Bounded $H_{\infty}$ synchronization and state estimation for discrete timevarying stochastic complex networks over a finite horizon," IEEE Transactions on Neural Networks, vol. 22, no. 1, pp. 145-157, 2011.

[21] H. Huang, G. Feng, and J. Cao, "Robust state estimation for uncertain neural networks with timevarying delay," IEEE Transactions on Neural Networks, vol. 19, no. 8, pp. 1329-1339, 2008.

[22] X. Liu and J. Cao, "Robust state estimation for neural networks with discontinuous activations," IEEE Transactions on Systems, Man, and Cybernetics B, vol. 40, no. 6, pp. 1425-1437, 2010.

[23] H. Bao and J. Cao, "Robust state estimation for uncertain stochastic bidirectional associative memory networks with time-varying delays," Physica Scripta, vol. 83, no. 6, Article ID 065004, 2011.

[24] H. Bao and J. Cao, "Delay-distribution-dependent state estimation for discrete-time stochastic neural networks with random delay," Neural Networks, vol. 24, no. 1, pp. 19-28, 2011.

[25] H. Huang, G. Feng, and J. Cao, "Guaranteed performance state estimation of static neural networks with time-varying delay," Neurocomputing, vol. 74, no. 4, pp. 606-616, 2011.

[26] E. Fridman, U. Shaked, and V. Suplin, "Input/output delay approach to robust sampled-data $H_{\infty}$ control," Systems \& Control Letters, vol. 54, no. 3, pp. 271-282, 2005.

[27] N. Li, J. Hu, J. Hu, and L. Li, "Exponential state estimation for delayed recurrent neural networks with sampled-data," Nonlinear Dynamics, vol. 69, pp. 555-564, 2012.

[28] N. Li, Y. Zhang, J. Hu, and Z. Nie, "Synchronization for general complex dynamical networks with sampled-data," Neurocomputing, vol. 74, no. 5, pp. 805-811, 2011.

[29] H. Gao, J. Wu, and P. Shi, "Robust sampled-data $H_{\infty}$ control with stochastic sampling," Automatica, vol. 45, no. 7, pp. 1729-1736, 2009.

[30] B. Shen, Z. Wang, and X. Liu, "A stochastic sampled-data approach to distributed $H_{\infty}$ filtering in sensor networks," IEEE Transactions on Circuits and Systems, vol. 58, no. 9, pp. 2237-2246, 2011.

[31] B. Shen, Z. Wang, and X. Liu, "Sampled-data synchronization control of dynamical networks with stochastic sampling," . In press.

[32] D. Yue, E. Tian, and Q. Han, "A delay system method to design of event-triggered control of networked control systems," in Proceedings of the 50th IEEE Conference on Decision and Control and European Control Conference (CDC-ECC '11), pp. 1668-1673, Institute of Electrical and Electronics Engineers, Orlando, Fla, USA, December 2011.

[33] S. Hu and D. Yue, "Event-triggered control design of linear networked systems with quantizations," ISA Transactions, vol. 51, pp. 153-162, 2012.

[34] S. Hu and D. Yue, "L2-gain analysis of event-triggered networked control systems: a discontinuous lyapunov functionalapproach," International Journal of Robust and Nonlinear Control. In press.

[35] Z. Liu and Z. Chen, "Event-triggered average-consensus for multi-agent systems," in Proceedings of the 29th Chinese Control Conference (CCC '10), pp. 4506-4511, July 2010.

[36] D. V. Dimarogonas and K. H. Johansson, "Event-triggered control for multi-agent systems," in Proceedings of the 48th IEEE Conference on Decision and Control Held Jointly with 28th Chinese Control Conference (CDC/CCC '09), pp. 7131-7136, Shanghai, China, December 2009.

[37] D. V. Dimarogonas and E. Frazzoli, "Distributed event-triggered control strategies for multiagent systems," in Proceedings of the 47th Annual Allerton Conference on Communication, Control, and Computing (Allerton'09), pp. 906-910, Monticello, Ill, USA, October 2009.

[38] D. Yue, E. Tian, and Y. Zhang, "A piecewise analysis method to stability analysis of linear continuous/discrete systems with time-varying delay," International Journal of Robust and Nonlinear Control, vol. 19, no. 13, pp. 1493-1518, 2009.

[39] D. Yue, E. Tian, Y. Zhang, and C. Peng, "Delay-distribution-dependent stability and stabilization of TS fuzzy systems with probabilistic interval delay," IEEE Transactions on Systems, Man, and Cybernetics B, vol. 39, no. 2, pp. 503-516, 2009.

[40] C. Peng and Y.-C. Tian, "Delay-dependent robust stability criteria for uncertain systems with interval time-varying delay," Journal of Computational and Applied Mathematics, vol. 214, no. 2, pp. 480-494, 2008.

[41] Y. Liu, Z. Wang, and X. Liu, "Global exponential stability of generalized recurrent neural networks with discrete and distributed delays," Neural Networks, vol. 19, no. 5, pp. 667-675, 2006. 


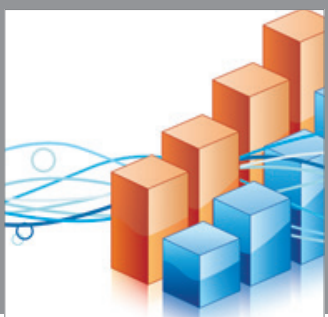

Advances in

Operations Research

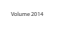

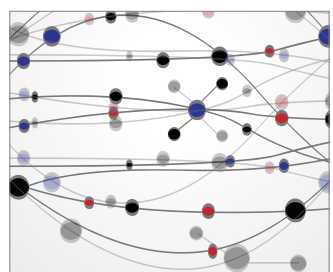

\section{The Scientific} World Journal
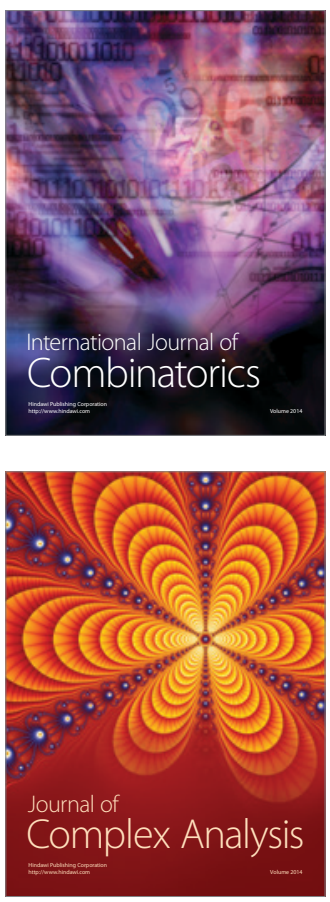

International Journal of

Mathematics and

Mathematical

Sciences
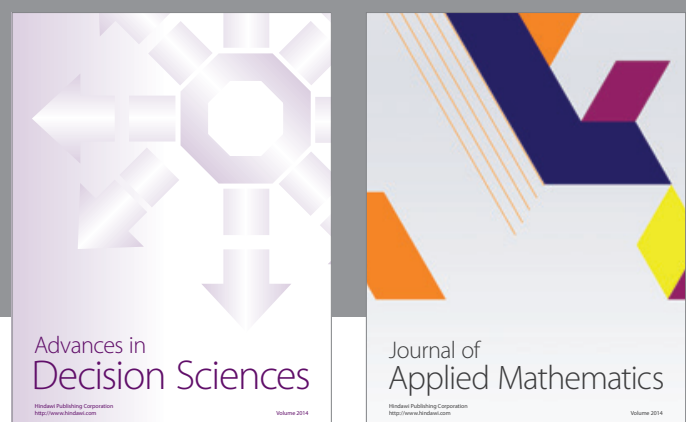

Journal of

Applied Mathematics
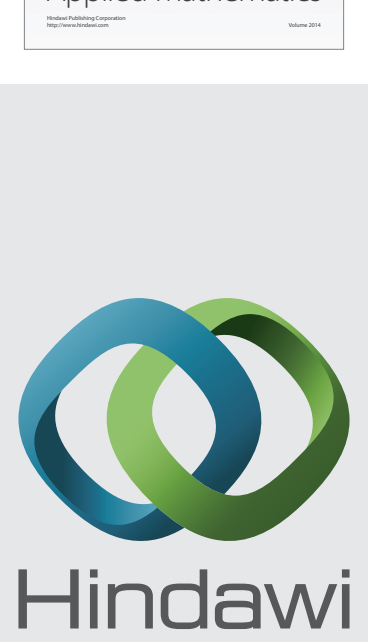

Submit your manuscripts at http://www.hindawi.com
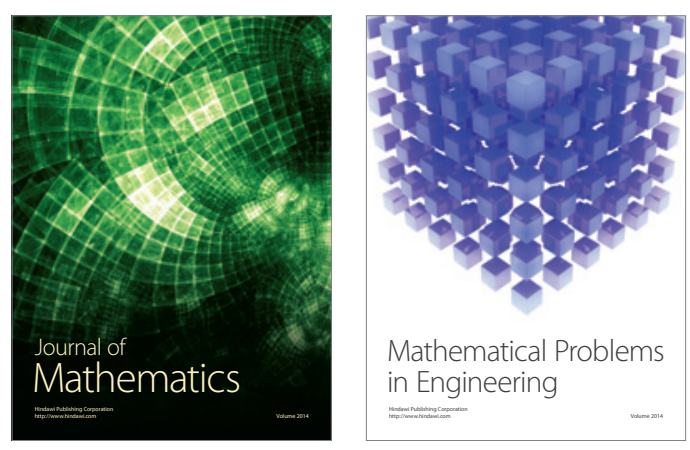

Mathematical Problems in Engineering
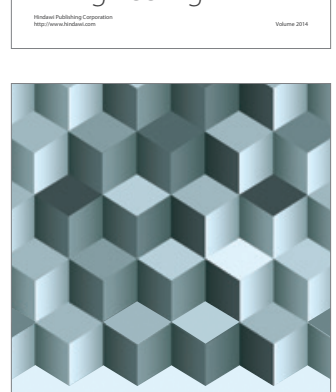

Journal of

Function Spaces
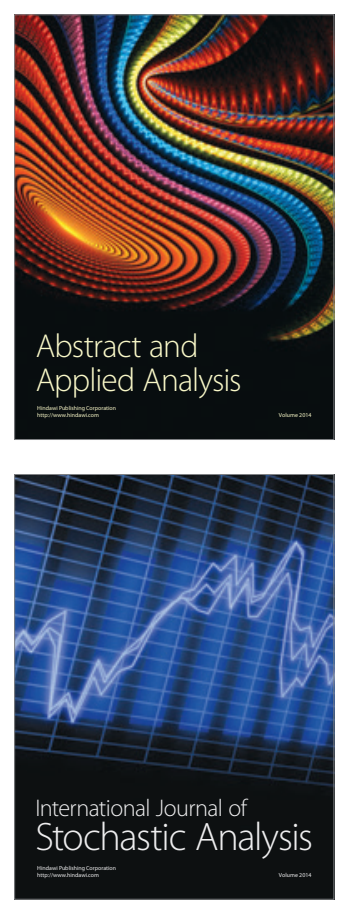

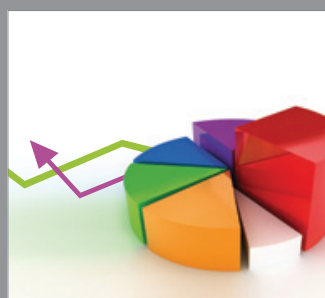

ournal of

Probability and Statistics

Promensencen
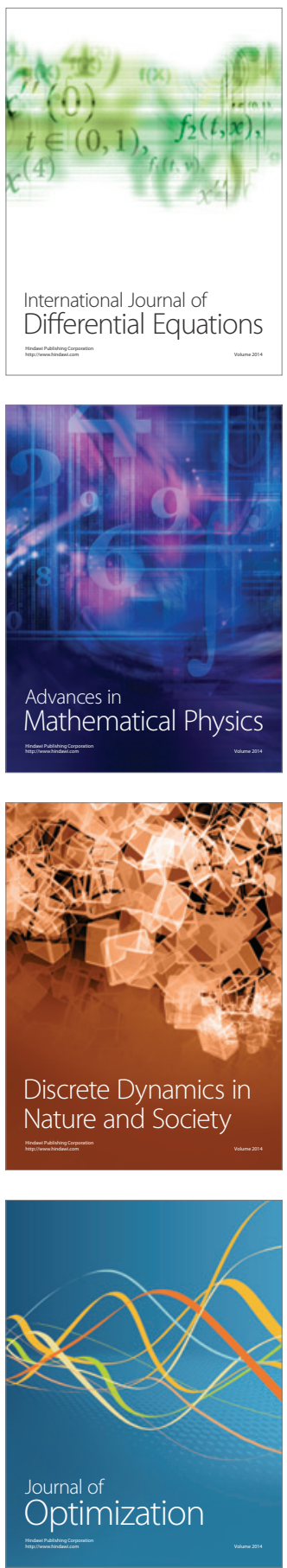